\title{
Nonlinear Aspects of Heat Pump Utilization
}

\author{
R. Najman
}

\begin{abstract}
This work attempts to answer the question: How much can we believe that the coefficient of performance provided by the manufacturer is correct, when a heat pump is required to face the real load coming from changes of temperature? The paper summarizes some basics of heat pump theory and describes the results of numerical models.
\end{abstract}

Keywords: heat pumps, thermal mass of an object, coefficient of performance.

\section{Introduction}

The idea of a heat pump is quite old, but nowadays we are confronted with the perspective of a potential energy crisis in the coming decades; people are starting to look for ways to lower their expenditure on domestic heating. A few years ago, when we started studies of this topic, only a few thousand heat pumps were installed in the Czech Republic, but the number has grown by $20-50 \%$ every year. Houses with additional thermal insulation and new insulation windows have spread even more. These installations have a profound impact on the performance of heat pumps.

\subsection{Basic principles of heat pumps}

Heat pumps use energy from a colder source and release it into a warmer ambience. Almost every refrigerator has one, so we have been living with heat pumps for a long time now.

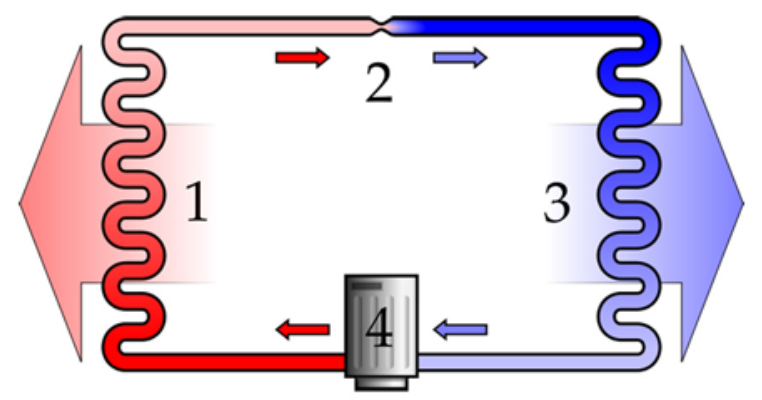

Legend

1 Condenser coil (hot side heat exchanger)

2 Expansion valve (gas expands, cools and liquifies)

3 Evaporator coil (cold side heat exchanger)

4 Compressor

Red $=$ Gas at high pressure and temperature

Pink $=$ Gas at high pressure and reduced temperature

Blue $=$ Liquid at low pressure and greatly reduced temperature

Light Blue $=$ Gas at low pressure and warmer temperature

Fig. 1: Basic idea of a heat pump $\left[{ }^{*} 1\right]$
To describe a heat pump correctly we need the following data: $C O P B_{\text {heating }}$, Source of heat, Target medium of heat transition

COP: (efficiency)

$$
C O P_{\text {heating }}=\frac{Q}{P} \text {. }
$$

Q Amount of heat transferred to hot reservoir

$C O P_{\text {heating }} \quad$ Coefficient of performance (for heating purposes)

$P \quad$ Dissipated work of the compressor

\subsection{Source and target of heat}

The three typical sources are air, water and soil. The targets are air or water. The systems are therefore referred to as air/air, air/water, water/air, water/water, soil/air and soil/water. For most technical and economic evaluation purposes, the target medium itself does not matter, only its temperature is important.

\subsubsection{Sources}

Air is the cheapest source for initial investment, but if you do not possess a source with stable temperature (such as warm air from some technological process) the COP is quite low, especially when temperatures outside hit minus values $\left({ }^{\circ} \mathrm{C}\right)$, or when you need output temperature above $40^{\circ} \mathrm{C}$.

Water is the "golden" middle way. It is a cheaper source than soil and its temperature is quite steady through the year, so achievable COP is quite good. Of course, use can be limited by unavailability of a usable water source. In most cases, the water source must be approved by the local authorities.

Soil is the most expensive, but surely the best source for COP. There are many technological ways to obtain heat from soil. The most common way is from drill holes, or from ground collectors. For new build- 
ings, a quite cheap and effective way is to use energy pilots (pilots of building foundations with integrated heat collectors).
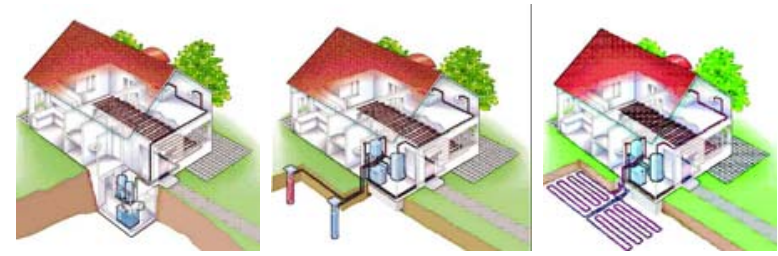

Fig. 2: Air, water, soil to water heat pumps [*2]

\section{Input values and equations}

\subsection{Subject for testing our mathematical model}

Our test subject is a house with a surface area of $400 \mathrm{~m}^{2}$ (surfaces with applicable thermal insulation).

Our model heat pump is a water/water type, source water $7^{\circ} \mathrm{C}$ (from a well), output $35^{\circ} \mathrm{C}$ for a screed floor. After making the first calculation of energy losses, the Stiebel Eltron WPW 7 heat pump seems to be right choice, in combination with some thermal insulation. With no thermal insulation we should use some larger model. Technical data of WPW7: $6.9 \mathrm{~kW}$ heat output at $35^{\circ} \mathrm{C}$ water and COP 5.2.

\subsection{Required heat output}

The heat output heat source is calculated according to the following model:

The indoor temperature $T_{v}$ is maintained by regulating temperature $T_{1}$.

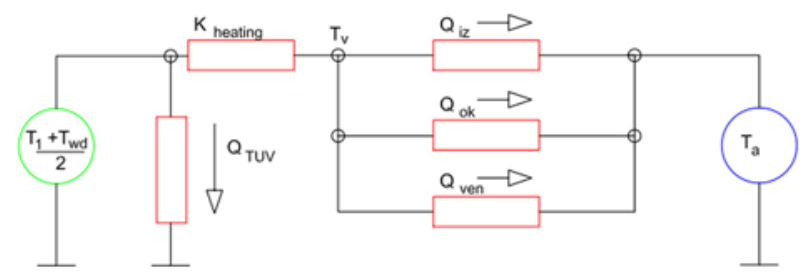

$T_{1} \quad$ highest heating temperature

$\left[{ }^{\circ} \mathrm{C}\right]$

$T_{w d} \quad$ lowest heating temperature

$\left[{ }^{\circ} \mathrm{C}\right]$

$T_{v} \quad$ indoor temperature

$K_{\text {heating }}$ constant representing efficiency of heat transfer

$Q_{\text {ven }} \quad$ heat losses through ventilation

$Q_{i z}$ heat losses through walls (insulated)

$Q_{o k}$. heat losses through windows

$Q_{T U V} \quad$ heat consumed on supply water

Fig. 3: Heat losses diagram
To calculate the heat losses we need to establish the thermal resistances

$$
\begin{array}{ll}
R_{t h v}=\frac{1}{\alpha_{v}}, & R_{t h z}=\frac{d}{\lambda_{z}}, \quad R_{t h i z}=\frac{d_{i z}}{1000 \cdot \lambda_{i z}} \\
R_{t h a}=\frac{1}{\alpha_{a}}, & R_{t h o}=\frac{1}{\lambda_{o}}
\end{array}
$$

Where:

$R_{t h v}$

indoor convection thermal resistance

$\left[\frac{\mathrm{K} \cdot \mathrm{m}^{2}}{\mathrm{~W}}\right]$

$\alpha_{v} \quad$ indoor heat transfer coefficient

$\left[\frac{\mathrm{W}}{\mathrm{K} \cdot \mathrm{m}^{2}}\right]$

$R_{t h z} \quad$ thermal resistance of wall

d wall width

$\left[\frac{\mathrm{K} \cdot \mathrm{m}^{2}}{\mathrm{~W}}\right]$ [m]

$\lambda_{z} \quad$ thermal conductivity of walls

$\left[\frac{\mathrm{W}}{\mathrm{K} \cdot \mathrm{m}}\right]$

$R_{\text {thiz }} \quad$ thermal resistance of walls

$\left[\frac{\mathrm{K} \cdot \mathrm{m}^{2}}{\mathrm{~W}}\right]$

$\lambda_{i z} \quad$ thermal conductivity of thermal insulation

$\left[\frac{\mathrm{W}}{\mathrm{K} \cdot \mathrm{m}}\right]$

$d_{i z} \quad$ thermal insulation width

$[\mathrm{mm}]$

$R_{t h a} \quad$ outdoor convection thermal resistance

$\left[\frac{\mathrm{K} \cdot \mathrm{m}^{2}}{\mathrm{~W}}\right]$

$\alpha_{a} \quad$ outdoor heat transfer coefficient

$\left[\frac{\mathrm{W}}{\mathrm{K} \cdot \mathrm{m}^{2}}\right]$

$R_{\text {tho }} \quad$ thermal resistance of windows

$\left[\frac{\mathrm{K} \cdot \mathrm{m}^{2}}{\mathrm{~W}}\right]$

$\lambda_{o} \quad$ thermal conductivity of windows $\quad\left[\frac{\mathrm{W}}{\mathrm{K} \cdot \mathrm{m}}\right]$

$$
\begin{aligned}
Q_{o k} & =S_{o k} \cdot \frac{T_{v}-T_{a}}{R_{t h v}+R_{t h o}+R_{t h a}} \\
Q_{i z} & =S \cdot \frac{T_{v}-T_{a}}{R_{t h v}+R_{t h z}+R_{t h i z}+R_{t h a}} \\
Q_{v e n} & =10 \cdot\left(T_{v}-T_{a}\right) \cdot o s-\text { empiric value }
\end{aligned}
$$

When using a heat pump, the consumption is given by CSN as follows:

(Heating to $35^{\circ} \mathrm{C}$, and then applying an additional source of heat. With other heat sources than the heat pumps it can be done in one step.)

$$
Q_{T U V}=\frac{o s \cdot 82 \cdot c_{p} \cdot(35-7)}{3600 \cdot 24}+\text { pom }
$$

Additional heating up to $55^{\circ} \mathrm{C}$ :

$$
\text { pom }=\frac{o s \cdot 82 \cdot c_{p} \cdot(55-35)}{3600 \cdot 24}
$$

os number of people

$c_{p} \quad$ specific heat capacity of water $[\mathrm{J} / \mathrm{kg} \cdot \mathrm{K}]$ 


\subsection{Heat pump in a thermal circuit}

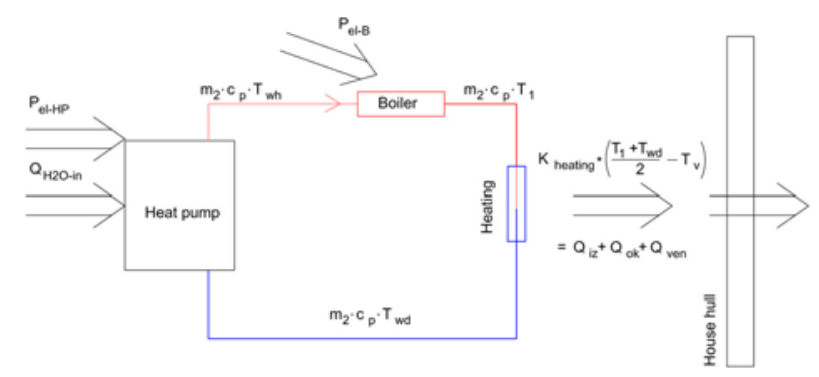

$Q_{\mathrm{H}_{2} \mathrm{O}-\mathrm{in}}$ heat taken from the source

$[\mathrm{W}]$

$P_{\text {el-HPB }}$ electricity consumption of HP

$m_{2} \quad$ mass flow the in heating circuit

$[\mathrm{kg} / \mathrm{s}]$

$T_{w h} \quad$ max. temp. of water in the HP

$P_{e l-B} \quad$ electricity consumption of a boiler

$[\mathrm{W}]$

(for others, see before)

Fig. 4: Thermal circuit

In the heat pump, the water is heated to Twh (approximately $35^{\circ} \mathrm{C}$ ), then it passes through the water boiler, where it can be heated to T1 (if necessary). In the screed floor, the temperature of the water goes down to Twd, and the cycle is repeated.

\subsection{Equations of the system}

$$
\begin{aligned}
C O P \cdot P_{\text {el-HP }} & =m_{2} \cdot c_{p} \cdot\left(T_{w h}-T_{w d}\right) \\
P_{e l-B} & =m_{2} \cdot c_{p} \cdot\left(T_{1}-T_{w h}\right) \\
Q_{\text {celk }} & =m_{2} \cdot c_{p} \cdot\left(T_{1}-T_{w d}\right) \\
Q_{\text {celk }} & =K_{\text {heating }} \cdot\left(\frac{T_{1}+T_{w d}}{2}-T_{v}\right)
\end{aligned}
$$

$Q_{\text {celk }}$ sum of all heat losses of the object [W]

This system of equations can be solved. The $m_{2}$ is chosen from the manufacturer's catalogue. The solutions are as functions of $d_{i z}$ and $T_{a}$ (from heat loss formulas).

\subsection{Thermal mass}

The nonlinear behavior of a heat pump (COP is a nonlinear function of the source and target temperature) indicates that the thermal mass of the object must be taken into account. Here I present the algorithm that allows us to estimate the impact of thermal masses on a defined thermal circuit.

The first step is to define the command function, which makes it easy for us to input the desired indoor temperature:

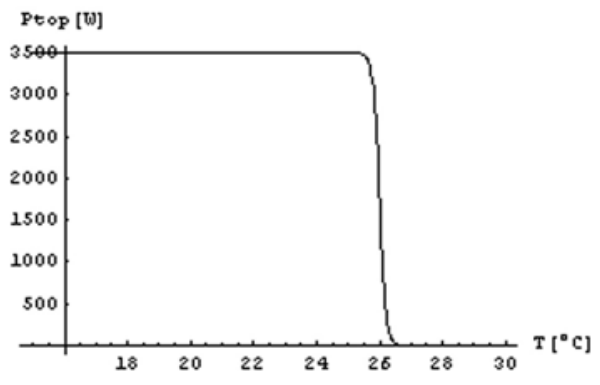

$P_{\text {top }}$ heating output $[\mathrm{W}]$

Fig. 5: Heating regulation

The following equations describe the thermal status of the system:

The wall:

$$
\rho_{s} \cdot c p_{s} \cdot \frac{\partial T_{s}(x, t)}{\partial t}=\lambda_{s} \cdot \frac{\partial^{2} T_{s}(x, t)}{\partial x^{2}}
$$

With this initial condition

$$
T_{s}(x, 0)=0
$$

and border conditions

$$
\begin{aligned}
& \frac{1}{\frac{1}{\alpha_{a}}+\frac{d_{i z}}{\lambda_{i z}}} \cdot\left(T_{a}(t)-T_{s}(0, t)\right)=-\lambda_{s} \cdot{\frac{\partial T_{s}(x, t)}{\partial x}}_{\mid x=0} \\
& \alpha_{v} \cdot\left(T_{s}(d, t)-T_{v}(d, t)\right)=-\lambda_{s} \cdot{\frac{\partial T_{s}(x, t)}{\partial x} \mid x=d}_{\mid x=d}
\end{aligned}
$$

Where:

$$
\begin{array}{lll}
\rho_{s} & \text { density } & {\left[\mathrm{kg} / \mathrm{m}^{3}\right]} \\
c p_{s} & \text { specific heat capacity } & {[\mathrm{J} / \mathrm{kg} \cdot \mathrm{K}]} \\
T_{s} & \text { temperature in the wall } & {\left[{ }^{\circ} \mathrm{C}\right]} \\
\lambda_{s} & \text { thermal conductivity of wall } & {[\mathrm{W} / \mathrm{K} \cdot \mathrm{m}]}
\end{array}
$$
(for others, see above)

Equations for air, adapted to fit better into Mathematica software:

$$
\begin{aligned}
& \rho_{v} \cdot c p_{v} \cdot \frac{\partial T_{v}(x, t)}{\partial t}=100 \cdot \frac{\partial^{2} T_{v}(x, t)}{\partial x^{2}}+ \\
& \frac{P_{t o p}\left(T_{v}(x, t)\right)-S \cdot \alpha_{v} \cdot\left(T_{v}(x, t)-T_{s}(x, t)\right)}{V}- \\
& \frac{\rho_{v} \cdot c p_{v} \cdot\left(T_{v}(x, t)-T_{a}\right)}{t_{v}}
\end{aligned}
$$

With the initial condition

$$
T_{v}(x, 0)=0
$$

and border conditions:

$$
\begin{aligned}
& \frac{\partial T_{v}(x, t)}{\partial x}=0_{\mid x \rightarrow 0} \\
& \frac{\partial T_{v}(x, t)}{\partial x}=0_{\mid x \rightarrow d}
\end{aligned}
$$


Where:

$\begin{array}{lll}T_{v} & \text { air temperature } & {\left[{ }^{\circ} \mathrm{C}\right]} \\ \rho_{v} & \text { air density } & {\left[\mathrm{kg} / \mathrm{m}^{3}\right]} \\ c p_{v} & \text { air specific heat capacity } & {[\mathrm{J} / \mathrm{kg} \cdot \mathrm{K}]} \\ S & \text { surface of walls } & {[\mathrm{S}]} \\ V & \text { indoor volume of air } & {\left[\mathrm{m}^{3}\right]} \\ P_{t o p} & \text { heating output } & {[\mathrm{W}]} \\ t_{v} & \text { time constant of ventilation } & {[\mathrm{s}]}\end{array}$

The solutions to the equations give heating output as a function of time. With sinusoid outdoor temperature in the graph below:

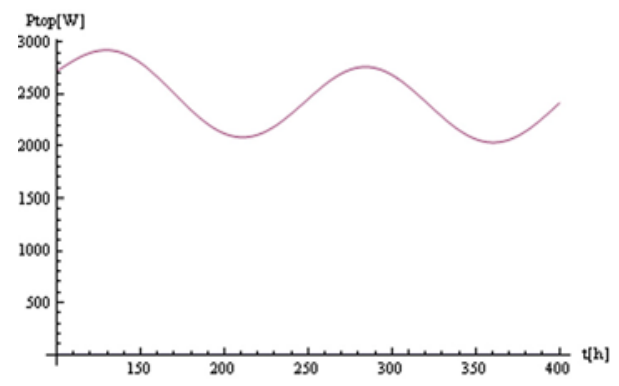

Fig. 6: Heating output

From the part of the solution where transient effects no longer apply, we extract a period of one day $P_{\text {top }}=a+b \cdot \sin (\omega t+\varphi)$.

If such heat loses are implemented into the heat pump model described above, we can obtain the dependencies of the variables needed to evaluate the effects of the thermal masses on the heat pump.

Where "red" is the power input of the heat pump, and "black" is the power input of water boiler.

Variant A: outdoor temperature -10 to $+10{ }^{\circ} \mathrm{C}$, sinus, without thermal mass

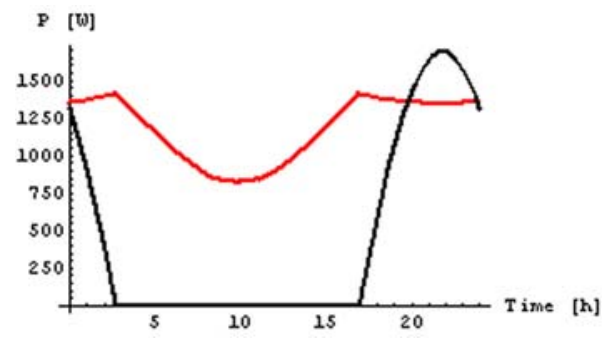

Fig. 7: Power inputs, variant A

Variant B: outdoor temperature -10 to $+10{ }^{\circ} \mathrm{C}$, sinus, with thermal mass

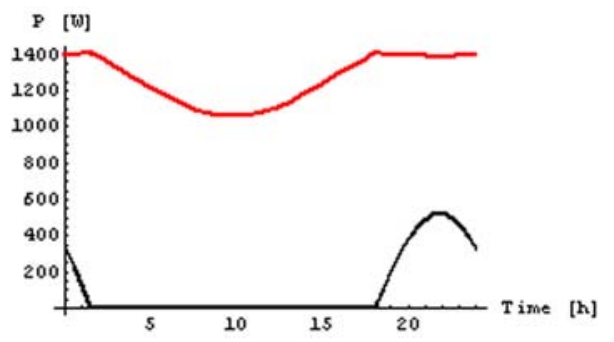

Fig. 8: Power inputs, variant $\mathrm{B}$
Variant C: Outdoor temperature $0{ }^{\circ} \mathrm{C}$

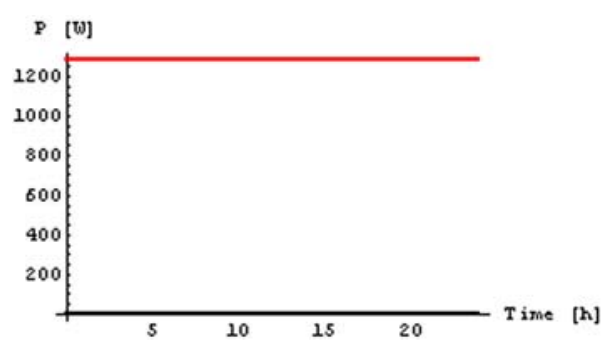

Fig. 9: Power inputs, variant $\mathrm{C}$

If the power inputs are integrated, there is little difference between Var. B (reality) and Var. C. (5.8 \%). However, since integrating the full thermal mass including model into our heat pump circuit model would increase the numerical flaws and greatly reduce the stability and reliability of the outcome, we chose from the models according to Var. A (no thermal mass) or Var. C (the thermal mass is so great, that it would negate all changes of temperature during one day). I have chosen to use a curve of input temperatures that it will simulate a system equivalent to variant $\mathrm{C}$. It is very close to reality, and can be solved more precisely with numerical methods in our system of equations.

\section{Output values of the heat pump circuit model$$
\text { (for } d_{i z}=50 \mathrm{~mm} \text { ) }
$$

If the outdoor temperature is stable, the input powers of the heat pump (red) and the water boiler (black) are as follows:

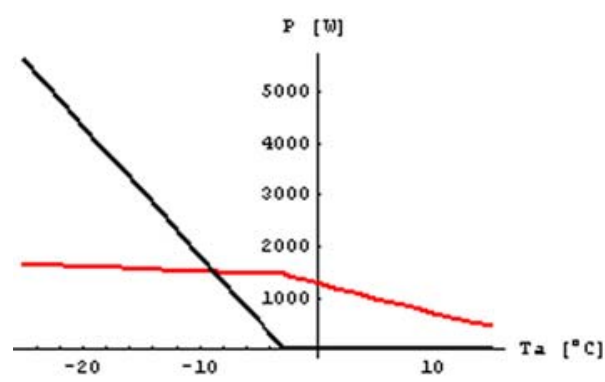

Fig. 10: Power inputs in stable conditions

For choosing the heating period of the year we take the long-term average temperature and compare it with $15^{\circ} \mathrm{C}$.

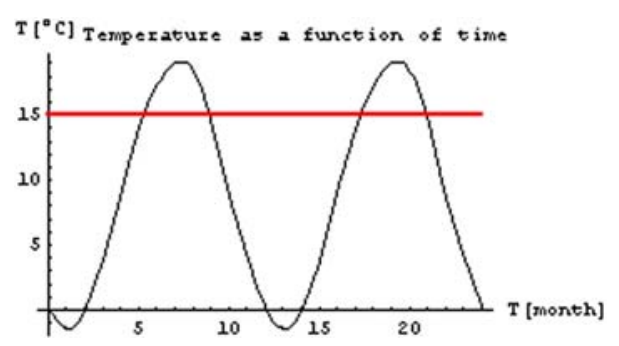

Fig. 11: Average temperature 
For the real effects on a heat pump, we add some oscillations to the outdoor temperature.

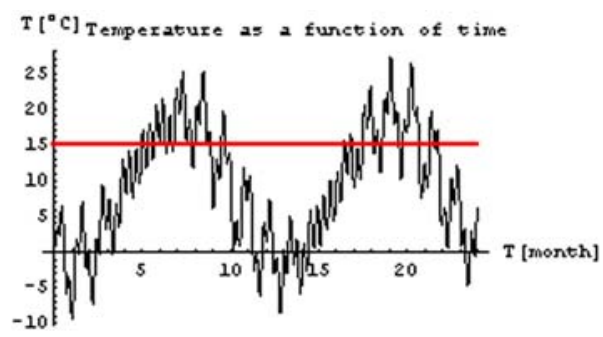

Fig. 12: Average temperature with oscillations

This gives us a good display of the consumed power of the heat pump and water boiler in the course of a year. It shows us that the heat pump (red) covers almost all heat losses. The water boiler (black) needs to be switched on only when there is a long period of cold weather.

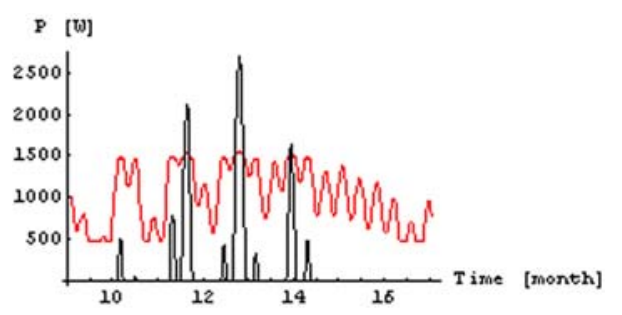

Fig. 13: Power consumptions in the course of a model year

We also obtain a very significant COP value (red):

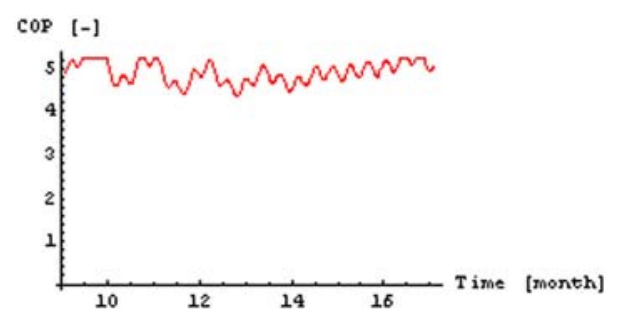

Fig. 14: COP in the course of model year

\section{Results}

With our input parameters the heat pump worked throughout the year with COP 4.82. The heat pump covers almost $100 \%$ of heat consumption with $5900 \mathrm{kWh}$ per year consumed for heating.

The simplified models give us different results:

The "merchant" model, which is often used by heat pump sellers gives us COP 5.2, and energy consumption of $5400 \mathrm{kWh}$. It calculates with an average temperature during the heating season.

The "normative" model, which uses $T_{a}=-15^{\circ} \mathrm{C}$ for the working parameters for heating gives us COP 2.5 and consumed power of circa $11000 \mathrm{kWh}$ per year.

This shows that it is worthwhile to investigate heat pumps utilization more deeply. Appropriate use of heat pumps should be considered on the basis of as much factual information as possible.

\section{Acknowledgement}

The research described in this paper was supervised by Doc. Dr. Jan Kyncl.

\section{References}

[1] http://en.wikipedia.org/wiki/Heat_pump

[2] Stiebel, E.: Heat Pump catalogue 2008.

Richard Najman

E-mail: najmaric@.fel.cvut.cz

Dept. of Electroenergetics

Czech Technical University

Technická 2, 16627 Praha, Czech Republic 